\title{
Assessment of Exclusive Breastfeeding Practice and Associated Factors among Mothers in West Shoa Zone, Oromia, Ethiopia
}

\author{
Kassa Mamo ${ }^{D},{ }^{1}$ Tizita Dengia, ${ }^{2}$ Abuzumeran Abubeker, ${ }^{2}$ and Eden Girmaye ${ }^{1}$ \\ ${ }^{1}$ Department of Midwifery, College of Medicine and Health Sciences, Ambo University, Ambo, Ethiopia \\ ${ }^{2}$ Department of Public Health, College of Medicine and Health Sciences, Ambo University, Ambo, Ethiopia \\ Correspondence should be addressed to Kassa Mamo; kasamam2001hu@gmail.com
}

Received 3 February 2020; Revised 17 July 2020; Accepted 27 July 2020; Published 12 August 2020

Academic Editor: Enrique Hernandez

Copyright (c) 2020 Kassa Mamo et al. This is an open access article distributed under the Creative Commons Attribution License, which permits unrestricted use, distribution, and reproduction in any medium, provided the original work is properly cited.

Background. The World Health Organization (WHO) recommends mothers worldwide to exclusively breastfeed infants for the child's first six months to achieve optimal growth, development, and health. Even though appropriate feeding practice is the most cost-effective intervention to reduce child morbidity and mortality, exclusive breastfeeding practices in developing countries are still low. Objective. The objective of the study was to assess exclusive breastfeeding practice and associated factors among mothers in West Shoa zone. Methods. Community-based cross-sectional study design was conducted from May to December 2018 in the West Shoa zone, Ethiopia, among 710 mothers with 6-9-month-old infants. The multistage stage sampling technique was employed. A pretested structured interviewer-administered questionnaire was used to collect the data. Epi Info version 7.1.2.0 was used to enter the data, and we transferred to SPSS version 25 for analysis. The association between factors and the exclusive breastfeeding were analyzed with bivariate and multivariate logistic regression. Result. A total of 710 women were included with a response rate of $97.9 \%$. The prevalence of unintended pregnancy was $38.7 \%$, and only $65.35 \%$ of the respondents reported that they have exclusively breastfed for the first six months of their infant's life. Marital status (AOR 2.467 (1.333-4.564)), ANC visit (AOR 2.562 (1.250-5.252)), pregnancy intentionality (AOR $4.727(3.217-6.945)$ ), postnatal care clinic attendance (AOR 3.373 (2.293-4.963)), and counseling on exclusive breastfeeding AOR 2.544 (1.239-5.225) were the factors associated with exclusive breastfeeding. Exclusive breastfeeding practice is still low and actions need to be taken like educating the community about the importance of exclusive breastfeeding using every accessible media. Maternal health service centers should provide counseling and education for women about breastfeeding.

\section{Introduction}

The World Health Organization recommends mothers to exclusively breastfeed infants for the child's first six months of life to achieve optimal growth, development, and health [1]. Babies who are breastfed exclusively for 6 months experience fewer illnesses because breast milk contains nutrients and substances that protect the baby from several infections and major childhood conditions, diarrhea, gastrointestinal tract infection, allergic diseases, diabetes, obesity, childhood leukemia and lymphoma, and inflammatory and bowel disease and it leads to improved cognitive development [2, 3].

According to Black et al. [4], suboptimal breastfeeding especially nonexclusive breastfeeding in the first six months of life results in 1.4 million deaths and $10 \%$ of disease burden in children younger than 5 years [5]. However, only $35 \%$ of infants worldwide are exclusively breastfed during the first four to six months of life and complementary feeding begins either too early or too late with foods that are often nutritionally inadequate and unsafe $[6,7]$.

Notwithstanding the fact that appropriate feeding practice is the most cost-effective intervention to reduce child morbidity and mortality [6], exclusive breastfeeding practices in developing countries are still low. Of approximately 56 million infants less than six months of age in developing countries, approximately 22 million are exclusively breastfed, while over 34 million children (60.7\%) are not. Eighty percent of these children who do not benefit from exclusive breastfeeding in developing countries live 
only in 29 countries. From these 29 countries, the 10 large countries including Ethiopia have two-thirds (over 21 million) of the approximate numbers of nonexclusively breastfed children in developing countries [8].

In Ethiopia, breastfeeding is a common practice, but a large proportion of mothers do not practice optimal breastfeeding. According to EDHS 2016 estimated, 58\% of under 6-month aged infants are exclusively breastfed. It is estimated that $74.1 \%$ of $0-1$-month-old infants, $64 \%$ of $2-3$ month-old infants, and $36 \%$ of $4-5$ infants were exclusively breastfed [9].

\section{Methods}

2.1. Study Design and Setting. A community-based crosssectional study design was employed. The study was conducted in west Shoa zone, Oromia, Ethiopia, from April to December 2018. West Shoa zone is one of the zones of the Oromia regional state. Ambo town is the capital of West Shoa. The zone consists of 22 districts and 583 kebeles. According to the 2007 Census conducted by the Central Statistics Agency of Ethiopia (CSA), this zone has a total population of 2,058,676, of whom 1,028,501 are men and $1,030,175$ women, with an area of $14,788.78$ square kilometers.

2.2. Sample Size Determination. All mothers whose infants are 6-9 months old and whose permanent residents in the area are for at least 6 months prior to conception were included in the study and mothers who are mentally disabled and unable to respond were excluded.

The sample size was calculated by using single population proportion formula using the prevalence of exclusive breastfeeding $34.1 \%$ from the study conducted in Bishoftu [10], 95\% confidence interval, and 5\% of margin error:

$$
\begin{aligned}
& \mathbf{n}=\frac{(\mathbf{Z}(\boldsymbol{\alpha} / 2))^{2} \mathbf{P}(1-\mathbf{P})}{\mathbf{d}^{2}} \\
& \mathbf{n}_{\mathbf{i}}=\frac{(1.96)^{2}(0.341)(1-0.341)}{(0.05)^{2}}=345 .
\end{aligned}
$$

Considering design effect and $10 \%$ nonresponse rate, the final sample size is 725 .

\subsection{Data Collection Instrument and Sampling Procedures.} A pretested structured interviewer-administered questionnaire was used to collect the data. Multistage sampling was used assuming homogeneity of the population in the study area. Twenty percent of the 22 districts, that is, 5 of them, were randomly selected using lottery method. Then, $20 \%$ of the kebeles in the districts were randomly selected using lottery method based on their number in order to ensure the representativeness of data. A structured questionnaire was developed after reviewing different literature in English language and translated into the local language. The questionnaire includes three parts which elicit information on the sociodemographic characteristics of the study participants, obstetric characteristics, and exclusive breastfeeding practice. A pretested structured interviewer-administered questionnaire was used to collect the data. The data were collected by face-to-face interviews by trained data collectors who were BSc midwives and fluent in the local dialect.

2.4. Data Quality and Analysis. The questionnaire was developed in English and translated into the local language Afan Oromo. Pretest was made on 5\% (37 mothers) of the total sample size. Training was provided to the data collectors on how to collect the data. All questionnaires were checked for completeness, coded, and entered using Epi Info version 7.1.2.0 and transferred to SPSS version 25 software package for analysis. Descriptive statistics such as mean, percentage, and standard deviations were determined. Data were analyzed using SPSS version 25 statistical software. The possible association was observed with binary logistic regression. All variables which have less than a $p$ value of 0.05 in the binary logistic regression were entered to multivariate logistic regression model. $p$ values of $\leq 0.05$ were used to declare significant association. Crude and adjusted odds ratios with their $95 \%$ confidence intervals were determined.

\section{Results}

3.1. Sociodemographic Characteristics of the Study Participants. A total of 710 women were included in the study, making a response rate of $97.9 \%$. The age of the respondents ranged from 16 to 43 years with the mean and median age being $28.9( \pm 6.04)$ and 29 years, respectively. Majority of the respondents $(73.7 \%)$ were Oromo by ethnicity. Most of the respondents were married (90.1\%). Urban dwellers make up $56.8 \%$ of the total respondents (Table 1).

3.2. Obstetric Characteristics of Respondents. More than $58 \%$ of women have at least one ANC visit in their most recent pregnancy. $36.2 \%$ the respondents are primiparous. Majority of the women have given birth in health institutions while $644(90.7 \%)$ women gave birth vaginally. Among the respondents, 275 (38.7\%) women reported that their most recent pregnancy was unintended. Among them, 128 (46.5\%) women said that their pregnancy was mistimed and the rest 147 (53.5\%) have had completely unwanted pregnancy (Table 2).

3.3. Exclusive Breastfeeding Practice. In this study the percentage of women who have exclusively breastfed accounts for $65.4 \%$ (464); the remaining 246 women reported that they did not breastfed exclusively (Figure 1). Among mothers who did not exclusively breastfed their infants for a minimum of 6-month duration, $48 \%$ (118) said that they never exclusively breastfed their child (Table 3 ).

3.4. Bivariate and Multivariate Analyses of Factors Associated with Exclusive Breastfeeding. On bivariate analysis besides intention of pregnancy, multiple factors were sought for 
TABLE 1: Sociodemographic characteristics of the study participants among women in west Shoa zone.

\begin{tabular}{|c|c|c|c|}
\hline & & Frequency & Percent \\
\hline \multirow{4}{*}{ Age of the mother } & $<18$ & 44 & 6.2 \\
\hline & $18-35$ & 532 & 74.9 \\
\hline & $>35$ & 134 & 18.9 \\
\hline & Total & 710 & 100.0 \\
\hline \multirow{5}{*}{ Ethnicity } & Oromo & 498 & 70.14 \\
\hline & Amhara & 178 & 25.1 \\
\hline & Gurage & 25 & 3.52 \\
\hline & Others & 9 & 1.3 \\
\hline & Total & 710 & 100.0 \\
\hline \multirow{3}{*}{ Marital status } & Married & 640 & 90.1 \\
\hline & Single & 70 & 9.9 \\
\hline & Total & 710 & 100.0 \\
\hline \multirow{4}{*}{ Religion } & Orthodox & 242 & 34.1 \\
\hline & Protestant & 433 & 61.0 \\
\hline & Muslim & 35 & 4.9 \\
\hline & Total & 710 & 100.0 \\
\hline \multirow{3}{*}{ Residence } & Urban & 403 & 56.8 \\
\hline & Rural & 307 & 43.2 \\
\hline & Total & 710 & 100.0 \\
\hline \multirow{6}{*}{ Educational status } & Illiterate & 138 & 19.4 \\
\hline & Can read and write with no formal education & 82 & 11.5 \\
\hline & Completed elementary/grades $1-8$ & 153 & 21.5 \\
\hline & Completed secondary school/grades $9-12$ & 184 & 25.9 \\
\hline & Above grade 12 & 153 & 21.5 \\
\hline & Total & 710 & 100.0 \\
\hline \multirow{6}{*}{ Monthly income } & Very low $(<600)$ & 181 & 25.5 \\
\hline & Low $(601-1500)$ & 128 & 18.0 \\
\hline & Average (1501-3500) & 167 & 23.7 \\
\hline & Above average (3501-5000) & 168 & 9.3 \\
\hline & High $(>5001)$ & 66 & 30.1 \\
\hline & Total & 710 & 100.0 \\
\hline
\end{tabular}

TABLE 2: Obstetric characteristics of the study participants among women in west Shoa zone.

\begin{tabular}{lccc}
\hline Variables & & Frequency & Percent \\
\hline \multirow{2}{*}{ ANC visit } & Yes & 432 & 60.8 \\
& No & 278 & 39.2 \\
\hline \multirow{2}{*}{ Parity } & Primiparous & 257 & 36.2 \\
& Multiparous & 453 & 63.8 \\
\hline \multirow{2}{*}{ Intention of pregnancy } & Wanted & 435 & 61.3 \\
& Mistimed & 128 & 38.7 \\
& Unwanted & 147 & \\
\multirow{2}{*}{ PNC attendance } & Yes & 441 & 62.1 \\
& No & 269 & 37.9 \\
\hline \multirow{2}{*}{ Place of delivery } & Home & 199 & 28.0 \\
& Health institution & 511 & 72.0 \\
\hline \multirow{2}{*}{ Mode of delivery } & Vaginal delivery & 644 & 90.7 \\
& Cesarean delivery & 66 & 9.3 \\
\hline
\end{tabular}

possible association with exclusive breastfeeding including age, marital status, place of residence, educational level, monthly income, ANC visit, mode of delivery, place of delivery, and sex of the child. Multivariate analysis identified that factors significantly associated with exclusive breastfeeding were intention of pregnancy, marital status, ANC

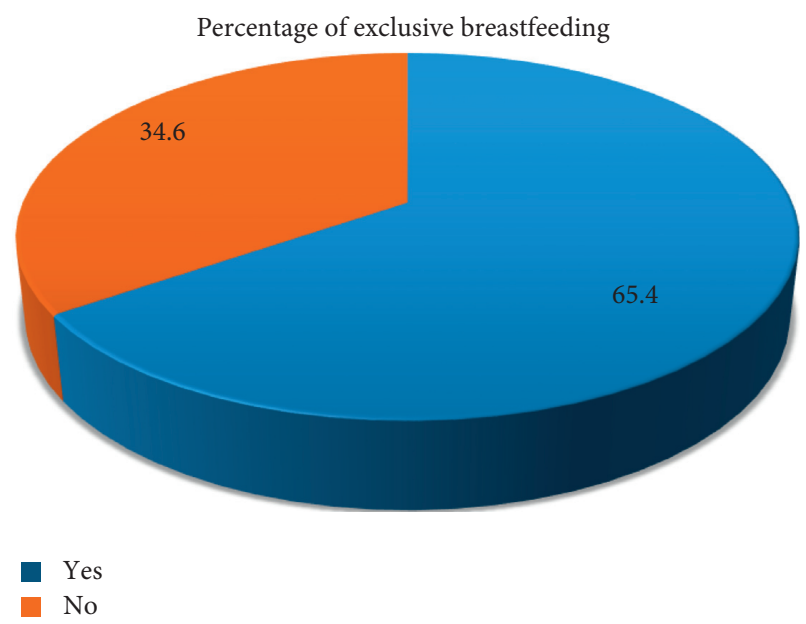

FIGURE 1: Percentage of exclusive breastfeeding among mothers in west Shoa zone.

visit, PNC attendance, and counseling on EBF. Women with intended pregnancy were more than four times likely to breastfeed exclusively compared to their counterparts (AOR 4.727, 95\% CI (3.217-6.945). Exclusive breastfeeding practice was higher among married women than single AOR 
TABLe 3: Duration of exclusive breastfeeding among women in west Shoa zone.

\begin{tabular}{lcc}
\hline Duration of exclusive breastfeeding & Frequency & Percent \\
\hline Never exclusively breasted & 118 & 48 \\
Less than 1 month & 11 & 4.5 \\
Between 1 and 2 months & 100 & 40.6 \\
Between 3 and 5 months & 17 & 6.9 \\
Total & 246 & 100 \\
\hline
\end{tabular}

TABLE 4: Bivariate and multivariate regression analysis of factors associated with exclusive breastfeeding among women in west Shoa zone.

\begin{tabular}{|c|c|c|c|c|c|c|}
\hline \multirow{2}{*}{ Variables } & & \multicolumn{2}{|c|}{ Exclusive breastfeeding } & \multirow{2}{*}{ COR $(95 \% \mathrm{CI})$} & \multirow{2}{*}{ AOR (95\% CI) } & \multirow{2}{*}{$p$ value } \\
\hline & & Yes $(\%)$ & No $(\%)$ & & & \\
\hline \multirow{2}{*}{ Marital status } & Married & $440(62.0 \%)$ & $200(28.2 \%)$ & $4.217(2.504-7.100)$ & $2.467(1.333-4.564)$ & 0.004 \\
\hline & Single & $24(3.4 \%)$ & $46(6.5 \%)$ & 1.00 & 1.00 & \\
\hline \multirow{2}{*}{ Residence } & Urban & $321(45.2 \%)$ & $86(12.1 \%)$ & $4.176(3.009-5.797)$ & $* *$ & \\
\hline & Rural & $143(20.1 \%)$ & $160(22.5 \%)$ & 1.00 & & \\
\hline \multirow{2}{*}{ ANC visit } & Yes & $348(49.0 \%)$ & $84(11.8 \%)$ & $5.786(4.130-8.105)$ & $2.562(1.250-5.252)$ & 0.010 \\
\hline & No & $116(16.3 \%)$ & $162(22.8 \%)$ & 1.00 & & \\
\hline \multirow{2}{*}{ Intention of pregnancy } & Intended & $349(49.2 \%)$ & $86(12.1 \%)$ & $5.646(4.034-7.903)$ & $4.727(3.217-6.945)$ & 0.000 \\
\hline & Unintended & $115(16.2 \%)$ & $160(22.5 \%)$ & 1.00 & & \\
\hline \multirow{2}{*}{ PNC attendance } & Yes & $337(47.5)$ & $104(14.6 \%)$ & $3.623(2.618-5.015)$ & $3.373(2.293-4.963)$ & 0.000 \\
\hline & No & $127(17.9 \%)$ & $142(20.0 \%)$ & 1.00 & & \\
\hline \multirow{2}{*}{ Mode of delivery } & Home & $432(60.8)$ & $212(29.9 \%)$ & $2.165(1.300-3.605)$ & $* *$ & \\
\hline & Institutional & $32(4.5 \%)$ & $34(4.8 \%)$ & 1.00 & & \\
\hline \multirow{2}{*}{ Counseling on EBF } & Yes & $355(50.0 \%)$ & $90(12.7 \%)$ & $5.645(4.031-7.905)$ & $2.544(1.239-5.225)$ & 0.011 \\
\hline & No & $109(15.4 \%)$ & $156(22.0 \%)$ & 1.00 & & \\
\hline \multirow{2}{*}{ Sex of the child } & Male & $311(43.8 \%)$ & $138(19.4 \%)$ & $1.519(1.162-1.985)$ & $* *$ & \\
\hline & Female & $151(21.3 \%)$ & $104(14.6 \%)$ & 1.00 & & \\
\hline
\end{tabular}

No significant association on multivariate regression analysis.

2.467, 95\% CI (1.333-4.564)). Mothers who have ANC follow-up were 2.562 more likely to breastfeed their child compared to women who did not have follow-up (AOR 2.562, 95\% CI (1.250-5.252)). Mothers who have PNC attendance were more than 3 times likely to exclusively breastfeed their babies compared to mothers who did not have PNC attendance (AOR 3.373, 95\% CI (2.293-4.963)) (Table 4).

\section{Discussion}

The purpose of the study was to assess exclusive breastfeeding practice and associated factors. Among the study participants, 464 (65\%) have reported that they have exclusively breastfed their babies for the appropriate time. The finding indicated that the prevalence of EBF is comparable to a study conducted in Debre Markos town which is $60.8 \%$ [11] but greater than studies in other parts of the country such as Bahirdar (50.3\%) [12], Mecha (47.13\%) [13], Injibara (44\%) [14], and Axum (40.9\%) [15]. Compared to the studies conducted in different parts of the world, the result showed that it is higher, where only $26 \%$ of the mothers in Cameroon [16], 20\% in Nigeria [17], $48 \%$ in Ghana [18], $44.3 \%$ in Malaysia [19], and 36\% in Bangladesh [16] reported that they have exclusively breastfed their babies. Considering the time these studies conducted, it would make sense for the variations to be huge as the institutional delivery and healthseeking behaviors have been increasing over time. Yet, it is lower than a study conducted in Saudi Arabia (76.1\%) [20].

$38.7 \%$ of the pregnancies in the current study were unintended. Among them, 128 (46.55\%) women stated that their pregnancy was mistimed and 147 (53.45\%) women reported it was unwanted. It is comparable to studies in Ethiopia like Hosanna (34\%) [21], Ganji (36.5\%) [22], Harar (33.3\%) [23], and Damot Gale (42.4\%) [24]; however, it is higher than studies in Kersa (27.9\%) [25], Dessie (21.5\%) [26], and Gelemso (27.1\%) [27] but less than the finding from Adigrat (69.5\%) [28]. It is less than a study conducted in Brazil which was 55.4\% [29].

Pregnancy intentionality was independently associated with exclusive breastfeeding. The odds of EBF among women who have intended pregnancy were four times higher than the odds in women who have unintended pregnancy (AOR 4.727, 95\% CI (3.217-6.945)). In line with the current study, a cross-sectional study from Hosanna has reported that planned pregnancies were more likely to be breastfed than their counterparts [21]. Similar findings were reported from other studies too, where unintended pregnancy does not only affect breastfeeding but also the prenatal care uptake during their pregnancy, and when the pregnancy is unwanted or mistimed, they either will initiate untimely or will not continue breastfeeding [30, 31]. Unintended 
pregnancy has also been associated with adverse pregnancy outcomes [32, 33]; correspondingly, reports indicated that timely wanted pregnancies are more likely to lead to breastfeeding initiation and longer duration [34]. This implies the importance of the provision of effective contraception to prevent unintended pregnancy which in turn affects child health and development.

The odds of EBF among women who did get counseling on exclusive breastfeeding were two times higher than the odds in women who did not get counseling on exclusive breastfeeding (AOR 2.544, 95\% CI (1.239-5.225)). In line with other studies, women who have ANC follow-up (AOR 2.562, 95\% CI (1.250-5.252)) and PNC (AOR 3.373, 95\% CI (2.293-4.963)) follow-up were more likely to exclusively breastfeed than their counterparts [13] [27]. Different studies reported that counseling on exclusive breastfeeding and infant feeding during their ANC follow-up and PNC follow-up has a strong association with exclusive breastfeeding $[11-13,35,36]$, which indicates the importance of effective counseling during prenatal and postnatal follow-up.

Marital status was found to be associated with EBF; married mothers were more than two times likely to exclusively breastfeed than single mothers (AOR 2.467, 95\% CI (1.333-4.564)). A similar finding has reported that nonexclusive breastfeeding was more likely to be practiced by mothers who were not married than married counterparts $($ AOR $(95 \% \mathrm{CI})=2.6(1.1,6.0))$ [37] that might be related to the fact that responsibility-sharing among family members could help the practice of exclusive breastfeeding.

\section{Conclusion}

$64.5 \%$ of women have practiced exclusive breastfeeding. ANC follow-up, PNC attendance, counseling, and pregnancy intentionality were factors associated with exclusive breastfeeding. Mothers should be counseled on the importance of exclusive breastfeeding during their ANC follow-up and postnatal visits. The study might suffer from limitations like information and recall biases to different variables which may result in under- or overestimation of exclusive breastfeeding practice. The nature of the study design could prevent an establishment of a cause-effect relationship between the factors and the dependent variable (EBF).

\section{Abbreviations}

AOR: Adjusted odds ratio

DHS: Demographic and health survey

CI: Confidence interval

EDHS: Ethiopian demographic and health survey

EBF: Exclusive breastfeeding

NGOs: Nongovernmental organizations

SSA: Sub-Saharan Africa

WHO: World Health Organization.

\section{Data Availability}

The data used in this study are available on request from the corresponding author.

\section{Ethical Approval}

Ethical approval was obtained from the Research review and ethics Committee of Ambo University, College of Medicine and Health Sciences (Reference no. CMHS/R-MW/04/17).

\section{Consent}

The purpose and importance of the research were explained to each of the study participants and the data were collected after a full-informed verbal consent was obtained, and they ensured their agreement by ticking on the [YES] checkbox on the information sheet and the data collectors had to make sure of that.

\section{Conflicts of Interest}

The authors declare that they have no conflicts of interest.

\section{Authors' Contributions}

Tizita Dengia and Abuzumeran Abubeker have initiated the research concept, developed the research proposal, and participated in analysis, report writing of the result, and drafted the manuscript. Kassa Mamo and Eden Girmaye have participated in data analysis and interpretation and writing the drafted manuscript. The authors read and approved the final manuscript.

\section{Acknowledgments}

The authors would like to express their gratitude to data collectors, supervisors, study participants, and west Shoa zone Administration Health Bureau for all their respective contribution for the realization of this study.

\section{Supplementary Materials}

The questionnaire used in this study. (Supplementary Materials)

\section{References}

[1] M. S. Kramer, The Optimal Duration of Exclusive Breastfeeding (Review), The Cochrane Library, London, UK, 2009.

[2] M. S. Noor Hafizan, T. Zainab, and R. Sutan, "Socio-demographic factors associated with duration of exclusive breastfeeding practice among mothers in east Malaysia," IOSR Journal of Nursing and Health Science, vol. 3, no. 1, pp. 52-56, 2014.

[3] WHO, "10 facts on child health," Journal of Pediatric Pharmacology, vol. 9, no. 3, p. 85, 2012.

[4] R. E. Black, L. H. Allen, Z. A. Bhutta et al., "Maternal and child undernutrition: global and regional exposures and health consequences," The Lancet, vol. 371, no. 9608, pp. 243-260, 2008.

[5] R. K. Ayisi and A. B. Wakoli, "Exclusive breastfeeding practice: its implication on nutrition status, growth and morbidity pattern among infants aged 0-6 months," Global Institute for Research \& Education, vol. 3, no. 1, pp. 254-258, 2014. 
[6] S. Patil and M. Yadavannavar, "Socio cultural factors affecting breast feeding practices and decisions in rural women," International Journal of Plant, Animal and Environmental Sciences, vol. 1, no. 2, pp. 46-50, 2011.

[7] L. Salami, "Factors influencing breastfeeding practices in edo state, Nigeria," African Journal of Food Agriculture Nutrition and Development, vol. 6, no. 2, 2006.

[8] UNICEF, Breastfeeding on the Worldwide Agenda: Findings from a Landscape Analysis on Political Commitment for Programmes to Protect, Promote and Support Breastfeeding, UNICEF, New York, NY, USA, 2013, http://www.unicef.org/ eapro/breastfeeding_on_worldwide_agenda.pdf.

[9] CSA, Ethiopia Demographic and Health Survey 2016, Central Statistical Agency, Addis Ababa, Ethiopia, 2016.

[10] Z. Kebede, "Determinants of optimum breastfeeding among mothers of child less than two years in Bishoftu town, east shewa zone of Oromia region, Ethiopia," Science Journal of Public Health, vol. 3, no. 4, pp. 544-551, 2015.

[11] G. Mekuria and M. Edris, "Exclusive breastfeeding and associated factors among mothers in Debre Markos, Northwest Ethiopia: a cross-sectional study," International Breastfeeding Journal, vol. 10, no. 1, 2015.

[12] A. M. Seid, M. E. Yesuf, and D. N. Koye, "Prevalence of exclusive breastfeeding practices and associated factors among mothers in Bahir Dar city, Northwest Ethiopia: a community based cross-sectional study," International Breastfeeding Journal, vol. 8, no. 1, 2013.

[13] T. G. Woldie, "Assessment of exclusive breast feeding practice and associated factors in Mecha district, north west Ethiopia," Science Journal of Public Health, vol. 2, no. 4, pp. 330-336, 2014.

[14] M. Taddele, "Exclusive breastfeeding and maternal employment in Ethiopia: a comparative cross- sectional study," International Journal of Nutrition and Food Sciences, vol. 3, no. 6, pp. 497-503, 2014.

[15] M. Alemayehu, "Factors associated with timely initiation and exclusive breast feeding among mothers of Axum town, northern Ethiopia," Science Journal of Public Health, vol. 2, no. 5, pp. 394-401, 2014.

[16] P. C. Joshi, M. R. Angdembe, S. K. Das, S. Ahmed, A. S. G. Faruque, and T. Ahmed, "Prevalence of exclusive breastfeeding and associated factors among mothers in rural Bangladesh: a cross-sectional study," International Breastfeeding Journal, vol. 9, no. 1, 2014.

[17] K. N. A. Pascale, N. J. Laure, and O. J. Enyong, "Factors associated with breast feeding as well as the nutritional status of infants (0-12) months: an epidemiological study in Yaounde, Cameroon," Pakistan Journal of Nutrition, vol. 6, no. 3, pp. 259-263, 2007.

[18] J. Danso, "Examining the practice of exclusive breastfeeding among professional working mothers in kumasi metropolis of Ghana," International Journal of Nursing, vol. 1, no. 1, p. 11, 2014.

[19] K. Tan, "Factors associated with exclusive breastfeeding among infants under six months of age in peninsular Malaysia," International Breastfeeding Journal, vol. 6, no. 1, p. 2, 2011.

[20] T. Amin, H. Hablas, and A. A. Al Qader, "Determinants of initiation and exclusivity of breastfeeding in Al hassa, Saudi Arabia," Breastfeeding Medicine, vol. 6, no. 2, pp. 59-68, 2011.

[21] B. Hamdela, G. Mariam, and T. Tilahun, "Unwanted pregnancy and associated factors among pregnant married women in Hosanna town, Southern Ethiopia," PLoS One, vol. 7, no. 6, 2012.

[22] F. T. Teshome, A. G. Hailu, and A. N. Teklehaymanot, "Prevalence of unintended pregnancy and associated factors among married pregnant women in Ganji woreda, west wollega Oromia region, Ethiopia," Science Journal of Public Health, vol. 2, no. 2, pp. 92-101, 2014.

[23] S. Worku and M. Fantahun, "Unintended pregnancy and induced abortion in a town with accessible family planning services: the case of Harar in eastern Ethiopia," Ethiopian Journal of Health Development, vol. 20, no. 2, pp. 79-83, 2007.

[24] N. R. Geda and T. K. Lako, "A population based study on unintended pregnancy among married women in a district in Southern Ethiopia," Journal of Geography and Regional Planning, vol. 4, no. 7, pp. 417-427, 2011.

[25] N. Kassa, Y. Berhane, and A. Worku, "Predictors of unintended pregnancy in Kersa, eastern Ethiopia, 2010," Reprod Health, vol. 9, no. 1, 2012.

[26] M. Arega, R.P. Surender, and Y. Ali, "Prevalence and associated factors of unintended pregnancy in Dessie city administration , east amhara, Ethiopia-Dec, 2014," Indian Journal of Medical Research, vol. 5, no. 4, 2016.

[27] F. Mohammed, A. Musa, and A. Amano, "Prevalence and determinants of unintended pregnancy among pregnant woman attending ANC at Gelemso general hospital, Oromiya region, East Ethiopia: a facility based cross-sectional study," BMC Womens Health, vol. 16, no. 56, 2016.

[28] A. Gessessew, "Abortion and unwanted pregnancy in Adigrat zonal hospital, tigray, north Ethiopia," African Journal of Reproductive Health, vol. 14, no. 3, pp. 183-188, 2010.

[29] M. M. Theme-Filha, M. L. Baldisserotto, A. C. S. A. Fraga, S. Ayers, S. G. Nogueira da Gama, and M. Do Carmo Leal, "Factors associated with unintended pregnancy in Brazil: cross-sectional results from the birth in Brazil national Survey, 2011/2012," Reprod Health, vol. 13, no. S 3, 2016.

[30] K. Kost and L. Lindberg, "Pregnancy intentions, maternal behaviors, and infant health: investigating relationships with new measures and propensity score analysis," Demography, vol. 52, no. 1, pp. 83-111, 2015.

[31] J. S. Taylor and H. J. Cabral, "Are women with an unintended pregnancy less likely to breastfeed?" The Journal of Family Practice, vol. 51, no. 5, pp. 431-436, 2002.

[32] D. Cheng, E. B. Schwarz, E. Douglas, and I. Horon, "Unintended pregnancy and associated maternal preconception, prenatal and postpartum behaviors," Contraception, vol. 79, no. 3, pp. 194-198, 2009.

[33] C. Marston and J. Cleland, "Do unintended pregnancies carried to term lead to adverse outcomes for mother and child? An assessment in five developing countries," Population Studies, vol. 57, no. 1, pp. 77-93, 2003.

[34] A. M. Stalcup, "Women's intentions for pregnancy and breastfeeding impact breastfeeding outcomes, implications \& opportunities (16F)," Obstetrics \& Gynecology 2018, vol. 221, no. 4, pp. 370-371, 2019.

[35] A. Earsido, W. Abebe, and N. Dereje, "Prevalence and determinants of exclusive breastfeeding practices among infants in hossana town, southern Ethiopia: a community based cross-sectional study," EC Gynaecology, vol. 4, no. 3, pp. 69-79, 2017.

[36] T. Tewabe, A. Mandesh, T. Gualu, G. Alem, G. Mekuria, and H. Zeleke, "Exclusive breastfeeding practice and associated factors among mothers in Motta town, East Gojjam zone, Amhara regional state, Ethiopia, 2015: a cross-sectional study," International Breastfeeding Journal, vol. 12, no. 1, 2015.

[37] G. Egata, Y. Berhane, and A. Worku, "Predictors of nonexclusive breastfeeding at 6 months among rural mothers in east Ethiopia: a community-based analytical cross-sectional study," International Breastfeeding Journal, vol. 8, no. 1, p. 8, 2013. 